\title{
Epac1 Blocks NLRP3 Inflammasome to Reduce IL-1 $\beta$ in Retinal Endothelial Cells and Mouse Retinal Vasculature
}

\author{
Youde Jiang, ${ }^{1}$ Li Liu, ${ }^{1}$ Elizabeth Curtiss, ${ }^{1}$ and Jena J. Steinle ${ }^{1,2}$ \\ ${ }^{1}$ Department of Anatomy and Cell Biology, Wayne State University School of Medicine, Detroit, MI, USA \\ ${ }^{2}$ Department of Ophthalmology, Wayne State University School of Medicine, Detroit, MI, USA \\ Correspondence should be addressed to Jena J. Steinle; jsteinle@med.wayne.edu
}

Received 1 November 2016; Revised 24 January 2017; Accepted 5 February 2017; Published 28 February 2017

Academic Editor: Ulrich Eisel

Copyright (C) 2017 Youde Jiang et al. This is an open access article distributed under the Creative Commons Attribution License, which permits unrestricted use, distribution, and reproduction in any medium, provided the original work is properly cited.

Inflammation is an important component of diabetic retinal damage. We previously reported that a novel $\beta$-adrenergic receptor agonist, Compound 49b, reduced Toll-like receptor 4 (TLR4) signaling in retinal endothelial cells (REC) grown in high glucose. Others reported that TLR4 activates high-mobility group box 1 (HMGB1), which has been associated with the NOD-like receptor 3 (NLRP3) inflammasome. Thus, we hypothesized that Epac1, a downstream mediator of $\beta$-adrenergic receptors, would block TLR4/HMGB1-mediated stimulation of the NLRP3 inflammasome, leading to reduced cleavage of caspase-1 and interleukin-1 beta (IL-1 $\beta$ ). We generated vascular specific conditional knockout mice for Epacl and used REC grown in normal and high glucose treated with an Epacl agonist and/or NLRP3 siRNA. Protein analyses were done for Epac1, TLR4, HMGB1, NLRP3, cleaved caspase1 , and IL-1 $\beta$. Loss of Epacl in the mouse retinal vasculature significantly increased all of the inflammatory proteins. Epacl effectively reduced high glucose-induced increases in TLR4, HMGB1, cleaved caspase-1, and IL-1 $\beta$ in REC. Taken together, the data suggest that Epacl reduces formation of the NLRP3 inflammasome to reduce inflammatory responses in the retinal vasculature.

\section{Introduction}

The role of inflammation and inflammatory mediators in the diabetic retina is becoming increasingly crucial for the development of pathology $[1,2]$. While a large number of potential inflammatory mediators are found in the diabetic rodent and human retina [3-5], the mechanisms to inhibit activation of these factors have proven more difficult to elucidate. Recent evidence has suggested that the diabetic environment (particularly type 2 diabetes) may provide the appropriate triggers to activate inflammasomes, specifically the NOD-like receptor 3 (NLRP3) inflammasome [6,7]. Inflammasomes are expressed in the cytosol and form in response to microbial or danger signals [8]. Activation of the inflammasome leads to the cleavage of caspase- 1 and activates interleukin-1 beta (IL-1 $\beta)[8,9]$. A number of factors can lead to the activation of an inflammasome, including reactive oxygen species, endoplasmic reticulum stress, and cationic fluxes [9]. Since most of these same factors are observed in the diabetic retina, it is probable that inflammasomes may contribute to diabetic retinal pathology. Reports have shown that acute glaucoma is associated with increased high-mobility group box 1 (HMGB1) leading to activation of the NLRP3 inflammasome [10]. Similarly, work in age-related macular degeneration has suggested that NLRP3 is upregulated in the retinal pigmented epithelial (RPE) cells [11]. Focusing on the diabetic retina, one study investigated RPE cells cultured in high glucose and showed an increase in NLRP3 inflammasome activation and IL-1 $\beta$ secretion in these cells [12]. However, RPE cells are not a predominant cell type affected in the diabetic retina. We have focused our work on retinal endothelial cells, as these cells are significantly affected in the diabetic retinal milieu.

We recently reported that a novel $\beta$-adrenergic receptor agonist, Compound 49b, could reduce Toll-like receptor 4 (TLR4) signaling in retinal endothelial cells (REC) grown in high glucose [13], suggesting that $\beta$-adrenergic receptors could regulate innate immune responses in the retina. $\beta$ Adrenergic receptors can signal through cAMP to activate protein kinase A (PKA) or exchange protein activated by cAMP (Epac). Both Epac1 and Epac2 have been localized in the retina [14], and we recently reported that Epacl can regulate IL-1 $\beta$ and inflammatory mediators in the retina [15]. 
Others have also suggested that Epacl can regulate inflammation in murine macrophages [16]. Similarly, work in Raw 264.7 macrophages showed that Epacl can regulate TLR4 [17].

Thus, in this study, we wanted to investigate whether Epac1 regulated the NLRP3 inflammasome to reduce IL-1 $\beta$ release in the retina. For this work, we used primary human retinal endothelial cells in culture, as well as vascular specific Epacl conditional knockout mice.

\section{Materials and Methods}

2.1. Vascular Specific Epac1 Knockout Mice. All animal procedures met the Association for Research in Vision and Ophthalmology requirements and were approved by the Institutional Animal Care and Use Committee of Wayne State University and conformed to $\mathrm{NIH}$ guidelines. Epacl floxed mice (B6; 129S2-Rapgef3 ${ }^{\text {tm1Geno/J }}$ mice) and B6 FVB$\mathrm{Tg}$ (cdh5-cre)7Mlia/J Cre mice were purchased from Jackson Laboratories. After 2 generations, the Epacl floxed mice were bred with cdh5-Cre mice to generate conditional knockout mice in which Epacl is eliminated in vascular endothelial cells [15]. At 3 months of age, Epac1 floxed and Epac1 Cre-Lox mice were used for experiments.

2.2. Retinal Endothelial Cells (REC). Primary human retinal endothelial cells (REC) were acquired from Cell Systems Corporation (CSC, Kirkland, Washington). Cells were grown in Cell Systems medium supplemented with microvascular growth factors (MVGS), $10 \mathrm{ug} / \mathrm{mL}$ gentamycin, and $0.25 \mathrm{ug} / \mathrm{mL}$ amphotericin B (Invitrogen, Carlsbad, CA) on attachment factor coated dishes. Once cells reached confluence, some dishes were moved to Cell Systems medium supplemented with D-glucose at $25 \mathrm{mM}$. Only cells prior to passage 6 were used. Cells were quiesced by incubating in high or normal glucose medium without MVGS for $24 \mathrm{~h}$ prior to experimental use.

2.3. Treatments. REC in high glucose $(25 \mathrm{mM})$ were transfected with NLRP3 siRNA (SMARTpool: ON-TARGETplus RAPGEF3 siRNA, Dharmacon, Lafayette, CO) or scrambled siRNA at a final concentration of $20 \mathrm{nM}$ using RNAiMAX transfection reagent according to the manufacturer's instructions. Twenty-four hours after transfection, some cells were treated with an Epacl agonist (8-CPT-2'-O-Me-cAMP) for an additional 2 hours at $10 \mathrm{uM}$. Analyses were done on REC samples after 3-4 days in normal or high glucose and 2 hours after Epacl stimulation.

2.4. Cytoplasmic Isolation for HMGB1. Some retinal lysates from the mice and REC were immediately processed for nuclear versus cytosolic extraction using the NE-PER kit (Thermo Scientific, Pittsburgh, PA). For these samples, extraction procedures were done according to the manufacturer's instructions. After extraction of each cellular portion, western blotting was done on only the cytoplasmic fraction.
2.5. Western Blotting. Whole retinal lysates from mice or cell culture lysates were collected in a lysis buffer containing protease and phosphatase inhibitors. Equal amounts of protein were separated onto a precast Tris-glycine gel (Invitrogen, Carlsbad, CA) and blotted onto nitrocellulose membrane. After blocking in TBST (10 mM Tris-HCl buffer, $\mathrm{pH} 8.0$, $150 \mathrm{mM} \mathrm{NaCl}$, and $0.1 \%$ Tween 20 ) and $5 \%(\mathrm{w} / \mathrm{v}) \mathrm{BSA}$, the membranes were treated with Epac1, TLR4, NLRP3, HMGB1, cleaved caspase-1 (Abcam, Cambridge, MA), or beta-actin (Santa Cruz Biotechnology, Santa Cruz, CA) primary antibodies followed by incubation with secondary antibodies labeled with horseradish peroxidase. Antigen-antibody complexes were detected by chemiluminescence reagent kit (Thermo Scientific, Pittsburgh, PA) and data was acquired using an Azure C500 (Azure Biosystems, Dublin, CA). Western blot data were assessed using Image Studio Lite software.

2.6. ELISA. IL-1 $\beta$ ELISA was completed according to the manufacturer's instructions with the exception of $120 \mathrm{ug}$ protein loaded into all wells, with the primary antibody incubated overnight.

2.7. Statistical Analyses. Nonparametric Kruskal-Wallis tests with Dunn's post hoc test were used for the cell culture data. One-way ANOVA with Student's Newman-Keuls post hoc test was used for animal work. $P<0.05$ was considered statistically significant.

\section{Results}

3.1. Loss of Epac1 in Endothelial Cells Increased Retinal Levels of TLR4 and Cytoplasmic HMGB1. Since we had reported that Compound $49 \mathrm{~b}$ could reduce TLR4 signaling [13], we wanted to determine whether this was through Epacl actions. We measured protein levels of TLR4 in retinal lysates from Epacl floxed or Epacl conditional knockout mice and found that loss of Epacl significantly increased TLR4 levels in the retina (Figure 1(a)) compared to the Epacl floxed mice. Since TLR4 often signals through HMGB1 to mediate inflammation $[18,19]$, we also measured cytosolic levels of HMGB1 in the mice. Loss of Epacl significantly increased cytosolic levels of HMGB1, suggesting that Epacl may be key to anti-inflammatory actions of Compound 49b.

3.2. NLRP3 and Cleaved Caspase-1 Are Increased in the Retina of Conditional Epac1 Knockout Mice. Others have reported that HMGB1 can be associated with NLRP3 inflammasomes in acute glaucoma [10]. Since Epacl reduced cytosolic HMGB1 levels, we wanted to determine whether Epac1 also could modulate NLRP3. We found that loss of Epacl in mouse retinal vasculature led to increased NLRP3 (Figure 2(a)) and cleaved caspase-1 (Figure 2(b)). Since activation of NLRP3 can activate cleaved caspase- 1 to increase IL- $1 \beta$ to mediate inflammation, our data showing increased NLRP3 and cleaved caspase-1 in Epacl conditional knockout mice suggest that Epacl may regulate these actions. We have previously reported that Epac1 Cre-Lox mice have increased IL-1 $\beta$ [15]. 


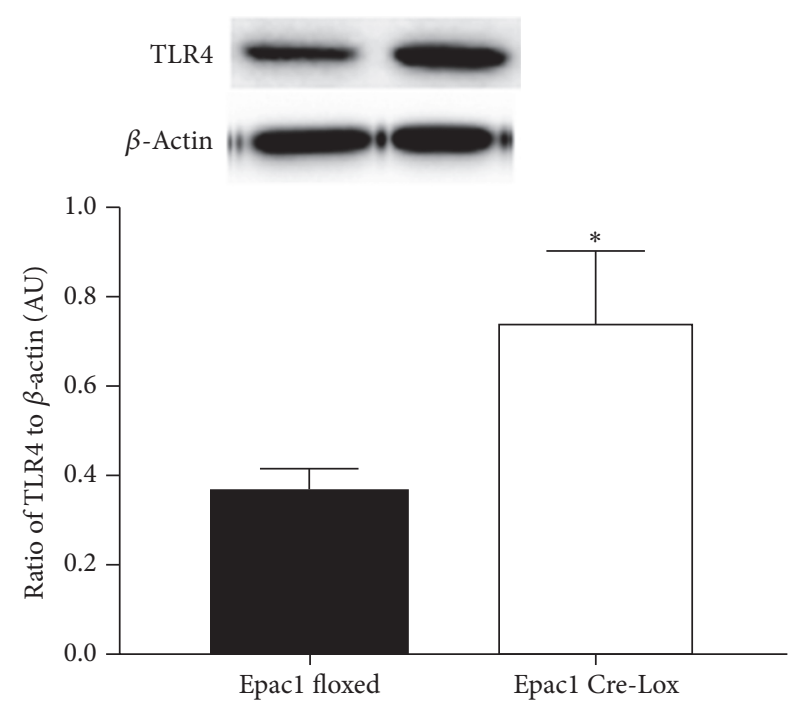

(a)

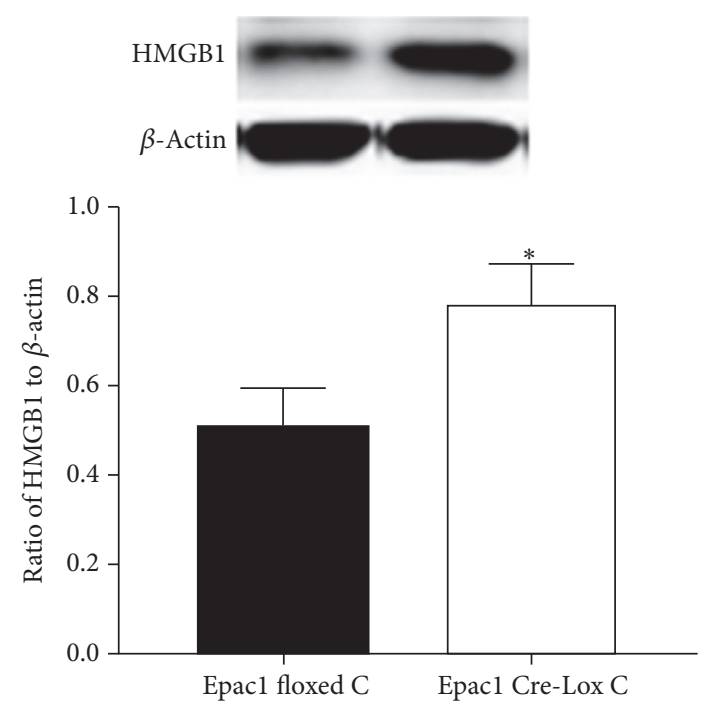

(b)

FIGURE 1: Loss of Epac1 increased TLR4 and cytosolic HMGB1. Western blot data from retinal lysates from Epac1 floxed and Epac1 Cre-Lox mice for TLR4 (a) and cytosolic HMGB1 (b). ${ }^{*} P<0.05$ versus Epacl floxed. $N=6$. Data are mean \pm SEM.

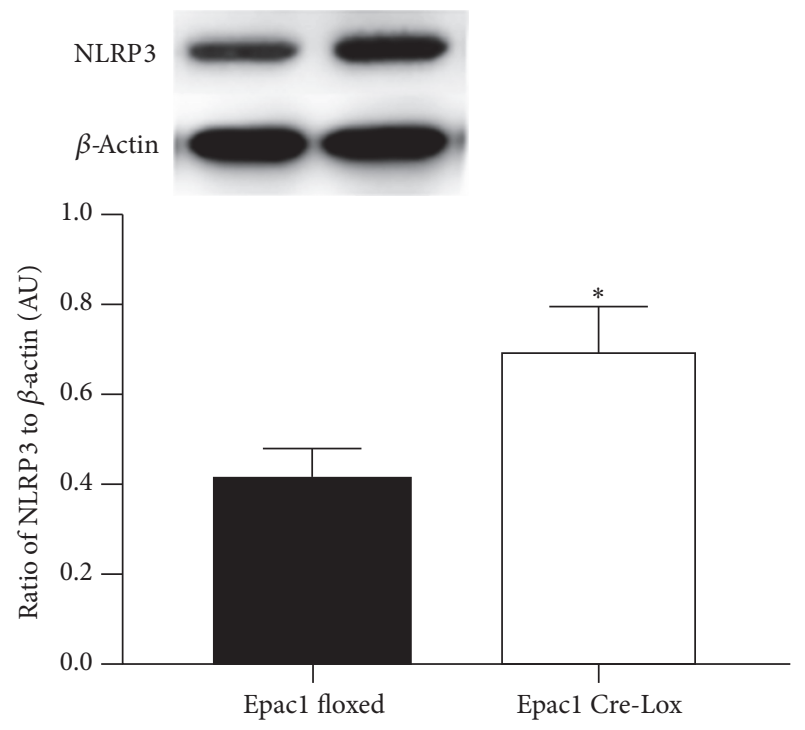

(a)

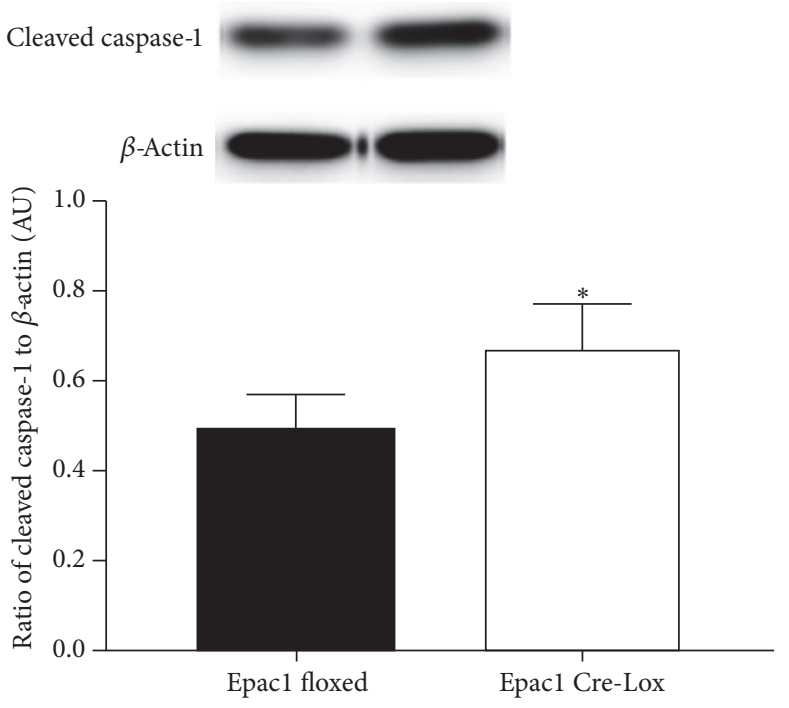

(b)

FIgURE 2: Epac1 reduces formation of the NLRP3 inflammasome and the cleavage of caspase-1. Western blot data from retinal lysates from Epac1 floxed and Epac1 Cre-Lox mice for NLRP3 (a) and cleaved caspase-1 (b). ${ }^{*} P<0.05$ versus Epac1 floxed. $N=6$. Data are mean \pm SEM.

3.3. An Epacl Agonist Can Significantly Reduce TLR4 and Cytoplasmic HMGB1 Levels in High Glucose Treated REC. To support our work in the conditional knockout mice, we also grew REC in normal and high glucose and measured TLR4 and HMGB1 levels after treatment with an Epacl agonist. Figure 3(a) shows that high glucose significantly reduced Epac1 levels, which were restored following Epacl agonist treatment. The Epacl agonist also significantly reduced high glucoseinduced increases in TLR4 (Figure 3(b)) and cytosolic HMGB1 (Figure 3(c)) compared to high glucose only. Use of the NLRP3 siRNA did not affect these levels, as it is likely that TLR4 and HMGB1 are upstream of the activation of the NLRP3 inflammasome [7, 8].

3.4. Epac1 and NLRP3 Can Reduce Cleaved Caspase-1 and IL$1 \beta$ Levels in REC Cultured in High Glucose. REC grown in high glucose have increased levels of NLRP3, cleaved caspase1 , and IL- $1 \beta$, which is reduced by treatment with an Epac1 agonist (Figures 4(a)-4(c)). These data agree with the data from mouse retinal lysates. Additionally, NLPR3 siRNA also significantly reduced cleaved caspase-1 (Figure 4(b)) and IL$1 \beta$ (Figure 4(c)) levels in REC. When the Epacl agonist and 


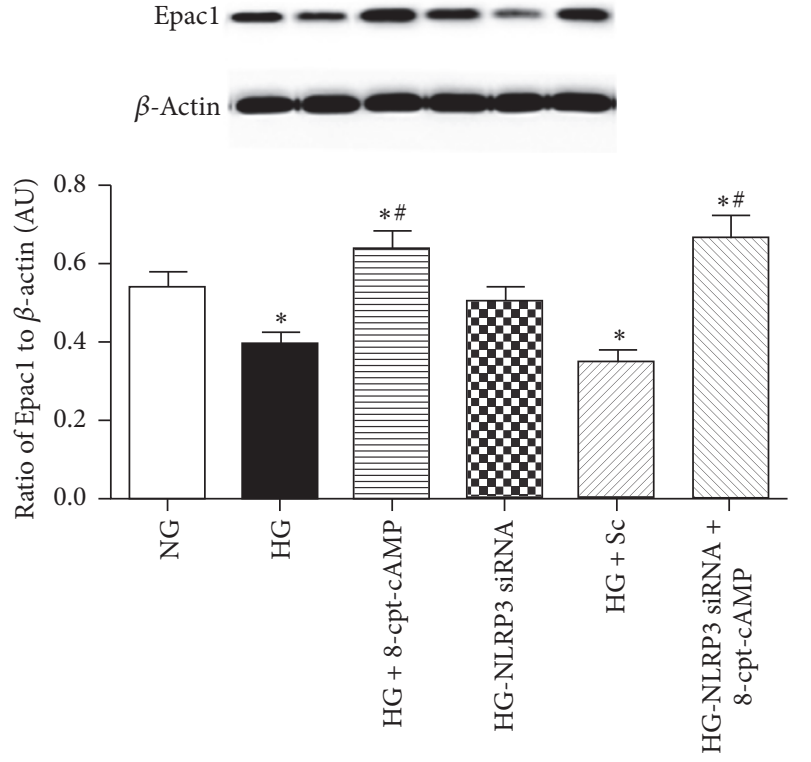

(a)

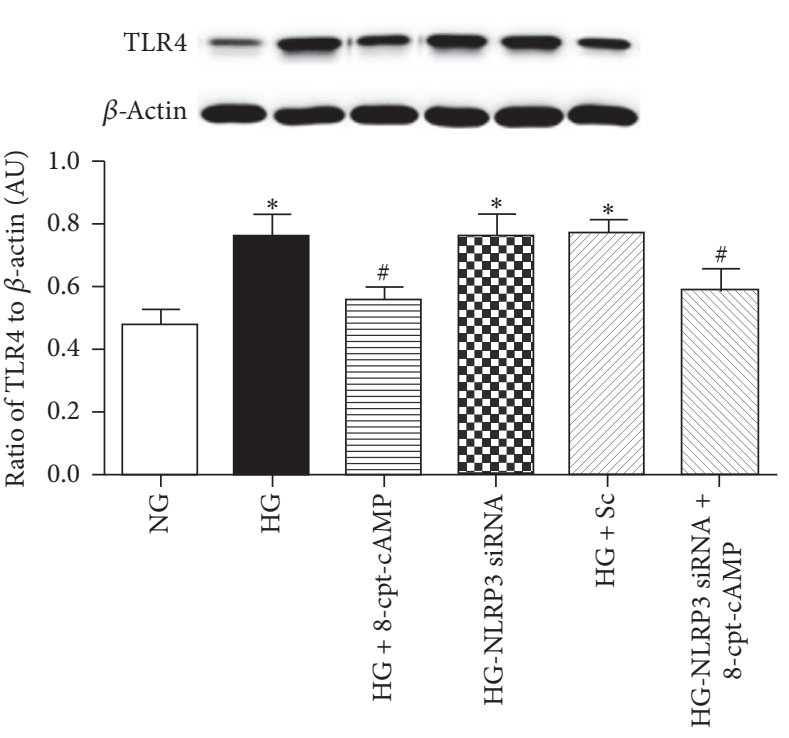

(b)

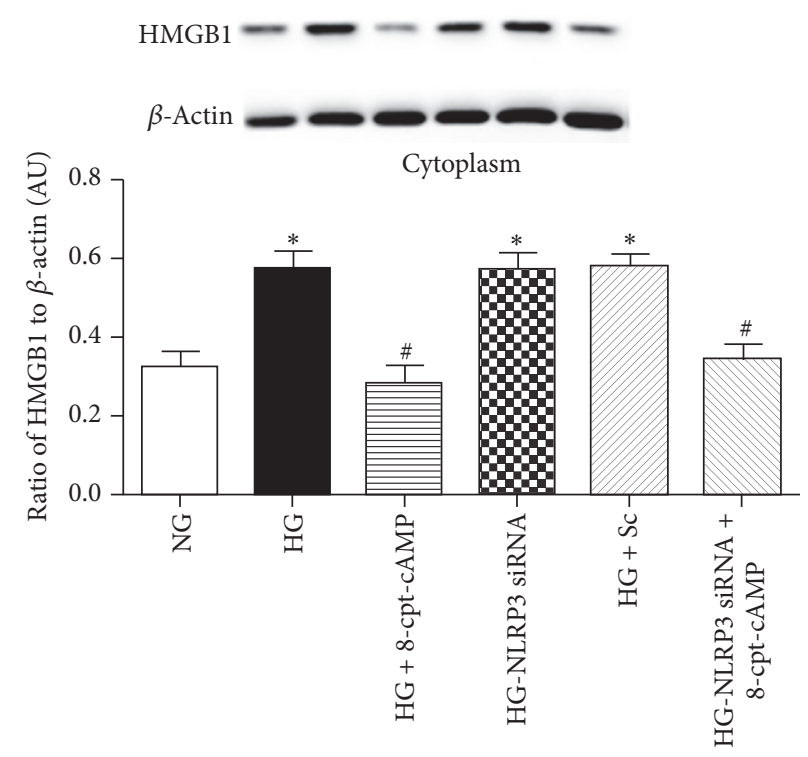

(c)

FIGURE 3: NLRP3 siRNA does not affect TLR4 and HMGB1 levels after Epac1 agonist treatment. Western blot data from REC grown in normal glucose (NG), high glucose (HG), high glucose + Epacl agonist (HG + 8-cpt-cAMP), high glucose + NLPR3 siRNA (HG + NLPR3 siRNA), high glucose + scrambled siRNA (HG + Sc), and high glucose + Epacl +NLRP3 siRNA (HG + NLRP3 siRNA + 8-cpt-cAMP) for Epacl (a), TLR4 (b), and cytosolic HMGB1 (c). $N=6$. Data are mean \pm SEM. ${ }^{*} P<0.05$ versus NG; ${ }^{*} P<0.05$ versus HG.

NLP3 siRNA were combined, the response was similar to the Epacl agonist or NLPR3 siRNA alone. Taken together with the mouse data, Epacl reduces NLRP3 levels, leading to reduced inflammation mediated by cleaved caspase- 1 and IL$1 \beta$.

\section{Discussion}

Few would argue that inflammation is a key component of the retinal damage associated with diabetes. While inhibition of specific inflammatory mediators has been effective in resolving some of the complications in rodents [4, 20, 21], increased understanding of upstream pathways may allow for more specific treatments or broad spectrum inhibition of the inflammatory process. Inhibition of receptor for advanced glycation end products (RAGE) has also proven effective in a rodent model of diabetes [22, 23]; however, other targets of TLR4 may also be effective. TLR4 can activate cytosolic HMGB1, leading to the initiation of inflammation [19, 24]. In acute glaucoma, HMGB1 activates the NLRP3 inflammasome, thus triggering the immune response [10]. We have previously reported that TLR4 signaling is increased in REC 


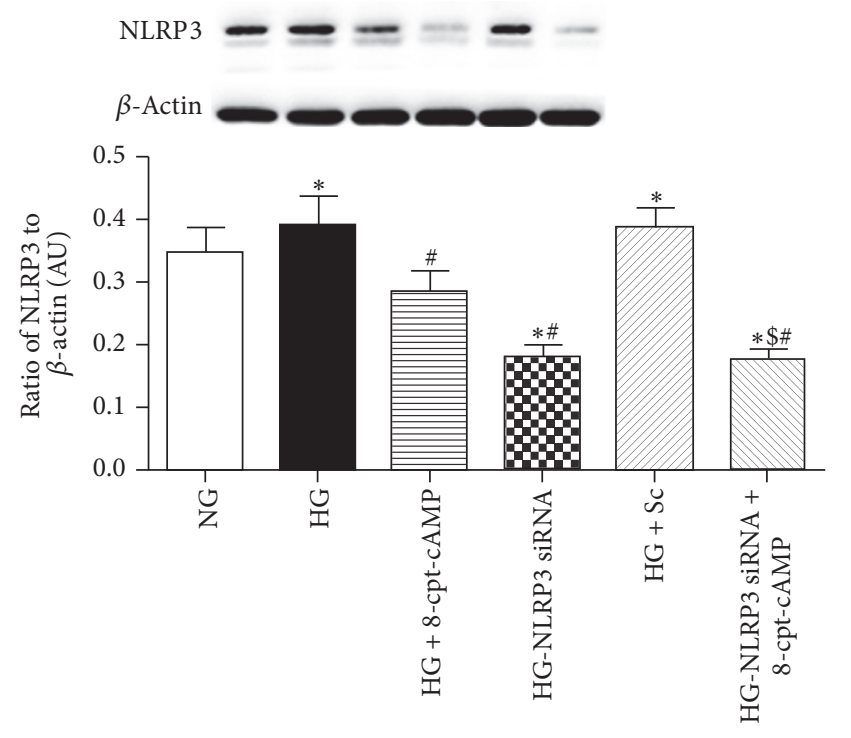

(a)

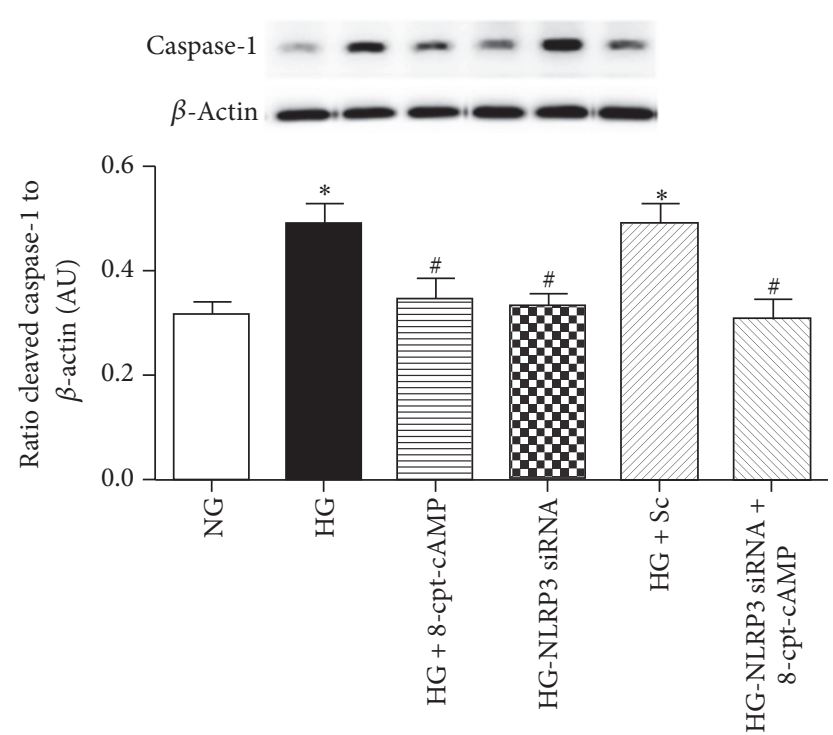

(b)

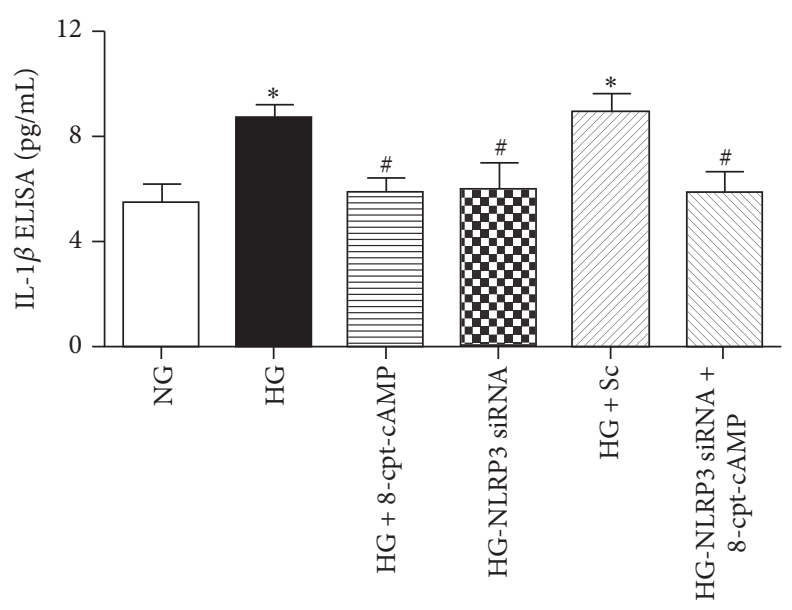

(c)

FIGURE 4: Epac1 and NLRP3 both can reduce cleavage of caspase-1 and IL-1 $\beta$. Protein analyses from REC grown in normal glucose (NG), high glucose (HG), high glucose + Epacl agonist (HG + 8-cpt-cAMP), high glucose + NLPR3 siRNA (HG + NLRP3 siRNA), high glucose + scrambled siRNA ( $\mathrm{HG}+\mathrm{Sc}$ ), and high glucose + Epacl agonist + NLRP3 siRNA (HG + NLRP3 siRNA + 8-cpt-cAMP) for NLRP3 (a), cleaved caspase-1 (b), and IL-1 $\beta$ (c). $N=6$ for NLRP3 and cleaved caspase-1; $N=5$ for ELISA for IL-1 $\beta$. Data are mean \pm SEM. ${ }^{*} P<0.05$ versus NG; ${ }^{\#} P<0.05$ versus $\mathrm{HG} ;{ }^{\$} \mathrm{P}<0.05$ versus $\mathrm{HG}+8$-cpt-cAMP.

grown in high glucose, which could be inhibited by Compound 49b [13]. In this work, we expanded those cell culture studies to demonstrate that Epacl, a downstream mediator of Compound 49b actions, is key to the reduction in TLR4 levels. Loss of Epacl in the mouse retinal vasculature also led to a significant increase in cytosolic HMGB1.

Because others have suggested that danger associated molecular patterns (DAMPs) can activate inflammasomes in type 2 diabetes [7], we wanted to investigate whether the cytosolic HMGB1 could stimulate formation of the NLRP3 inflammasome in the retina, as well as the role of Epacl in this activation. Data demonstrate that Epacl can inhibit NLRP3 levels, as well as the cleavage of caspase-1 in the retinal vasculature. We have already reported that loss of Epacl increased
IL-1 $\beta$ levels in the retina [15]. While there is little literature on Epac1 regulation of the NLRP3 inflammasome, there is work to support an anti-inflammatory role of Epacl in macrophages $[16,17]$.

To support our mouse retinal data, we also grew primary REC in normal and high glucose and found that an Epac1 agonist could significantly reduce both TLR4 and cytosolic HMGB1, which was not altered by NLRP3 siRNA, suggesting that both TLR4 and HMGB1 are upstream of NLRP3. However, cleaved caspase- 1 and IL- $1 \beta$ levels were not reduced by an Epacl agonist when cells were transfected with NLRP3 siRNA. Taken together, these data suggest that Epacl reduces TLR4 and HMGB1 to inhibit the NLRP3 inflammasome activation in the retinal vasculature (Figure 5). In future work, 


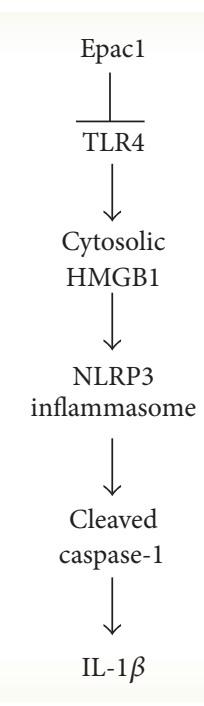

FIGURE 5: Schematic of the proposed regulation of the NLRP3 inflammasome by Epac1.

these studies will be expanded into the diabetic retina in vivo. Additionally, it is highly likely that other factors can also regulate the NLRP3 inflammasome in the diabetic retina, including thioredoxin interacting proteins (TXNIP) [6]. Interestingly, both NLRP3 and TXNIP knockout mice have reduced insulin resistance [25]. Thus, it will be interesting to investigate the role of Epacl in these pathways.

\section{Conclusions}

These studies demonstrate that loss of Epacl can increase inflammatory signaling in the retina, specifically TLR4/ HMGB1-mediated activation of the NLRP3 inflammasome and IL- $1 \beta$ secretion. TLR4 and HMGB1 are upstream of NLRP3 as they were unaffected by siRNA treatment in Epaclstimulated REC grown in high glucose. Mouse retinal samples showed increased inflammatory pathways in vascular specific Epacl conditional knockout mice. Epacl may serve as a novel broad spectrum anti-inflammatory factor for the retina through inhibition of the NLRP3 inflammasome.

\section{Competing Interests}

The authors declare that they have no competing interests regarding the publication of this paper.

\section{Acknowledgments}

This work was supported by R01EY022045 (Jena J. Steinle), P30EY04068 (PI: Hazlett), and an unrestricted grant to the Department of Ophthalmology from Research to Prevent Blindness (Kresge Eye Institute).

\section{References}

[1] J. Tang and T. S. Kern, "Inflammation in diabetic retinopathy," Progress in Retinal and Eye Research, vol. 30, no. 5, pp. 343-358, 2011.

[2] T. S. Kern, "Contributions of inflammatory processes to the development of the early stages of diabetic retinopathy," Experimental diabetes research, vol. 2007, Article ID 95103, 2007.

[3] L. Zheng, Y. Du, C. Miller et al., "Critical role of inducible nitric oxide synthase in degeneration of retinal capillaries in mice with streptozotocin-induced diabetes," Diabetologia, vol. 50, no. 9, pp. 1987-1996, 2007.

[4] S. F. Abcouwer, C.-M. Lin, S. Shanmugam, A. Muthusamy, A. J. Barber, and D. A. Antonetti, "Minocycline prevents retinal inflammation and vascular permeability following ischemiareperfusion injury," Journal of Neuroinflammation, vol. 10, article 149, 2013.

[5] J. A. Vincent and S. Mohr, "Inhibition of caspase-1/interleukin$1 \beta$ signaling prevents degeneration of retinal capillaries in diabetes and galactosemia," Diabetes, vol. 56, no. 1, pp. 224-230, 2007.

[6] D. De Nardo and E. Latz, "NLRP3 inflammasomes link inflammation and metabolic disease," Trends in Immunology, vol. 32, no. 8, pp. 373-379, 2011.

[7] R. W. Grant and V. D. Dixit, "Mechanisms of disease: inflammasome activation and the development of type 2 diabetes," Frontiers in Immunology, vol. 4, article 50, 2013.

[8] S. K. Vanaja, V. A. K. Rathinam, and K. A. Fitzgerald, "Mechanisms of inflammasome activation: recent advances and novel insights," Trends in Cell Biology, vol. 25, no. 5, pp. 308-315, 2015.

[9] I. N. Mohamed, T. Ishrat, S. C. Fagan, and A. B. El-Remessy, "Role of inflammasome activation in the pathophysiology of vascular diseases of the neurovascular unit," Antioxidants and Redox Signaling, vol. 22, no. 13, pp. 1188-1206, 2015.

[10] W. Chi, H. Chen, F. Li, Y. Zhu, W. Yin, and Y. Zhuo, "HMGB1 promotes the activation of NLRP3 and caspase- 8 inflammasomes via NF- $\kappa$ B pathway in acute glaucoma," Journal of Neuroinflammation, vol. 12, no. 1, article 137, 2015.

[11] Y. Wang, J. W. Hanus, M. S. Abu-Asab et al., "NLRP3 upregulation in retinal pigment epithelium in age-related macular degeneration," International Journal of Molecular Sciences, vol. 17, no. 1, article 73, 2016.

[12] H. Shi, Z. Zhang, X. Wang et al., "Inhibition of autophagy induces IL- $1 \beta$ release from ARPE-19 cells via ROS mediated NLRP3 inflammasome activation under high glucose stress," Biochemical and Biophysical Research Communications, vol. 463, no. 4, pp. 1071-1076, 2015.

[13] E. A. Berger, T. W. Carion, Y. Jiang et al., " $\beta$-Adrenergic receptor agonist, compound $49 \mathrm{~b}$, inhibits TLR4 signaling pathway in diabetic retina," Immunology and Cell Biology, vol. 94, no. 7, pp. 656-661, 2016.

[14] C. M. Whitaker and N. G. F. Cooper, "Differential distribution of exchange proteins directly activated by cyclic AMP within the adult rat retina," Neuroscience, vol. 165, no. 3, pp. 955-967, 2010.

[15] Y. Jiang, L. Liu, and J. J. Steinle, “Compound 49b regulates ZO1 and occludin levels in human retinal endothelial cells and in mouse retinal vasculature," Investigative Opthalmology \& Visual Science, vol. 58, no. 1, pp. 185-189, 2017.

[16] E.-Y. Moon, J.-H. Lee, J.-W. Lee, J.-H. Song, and S. Pyo, "ROS/Epacl-mediated Rap1/NF-kappaB activation is required for the expression of BAFF in Raw264.7 murine macrophages," Cellular Signalling, vol. 23, no. 9, pp. 1479-1488, 2011. 
[17] E.-Y. Moon, Y.-S. Lee, W. S. Choi, and M.-H. Lee, “Toll-like receptor 4-mediated cAMP production up-regulates B-cell activating factor expression in Raw264.7 macrophages," Experimental Cell Research, vol. 317, no. 17, pp. 2447-2455, 2011.

[18] J. R. Van Beijnum, W. A. Buurman, and A. W. Griffioen, "Convergence and amplification of toll-like receptor (TLR) and receptor for advanced glycation end products (RAGE) signaling pathways via high mobility group B1 (HMGB1)," Angiogenesis, vol. 11, no. 1, pp. 91-99, 2008.

[19] A. Tsung, S. Tohme, and T. R. Billiar, "High-mobility group box1 in sterile inflammation," Journal of Internal Medicine, vol. 276, no. 5, pp. 425-443, 2014.

[20] A. M. Joussen, V. Poulaki, N. Mitsiades et al., "Nonsteroidal anti-inflammatory drugs prevent early diabetic retinopathy via TNF-alpha suppression," The FASEB Journal, vol. 16, no. 3, pp. 438-440, 2002.

[21] T. S. Kern and R. L. Engerman, "Pharmacological inhibition of diabetic retinopathy: aminoguanidine and aspirin," Diabetes, vol. 50, no. 7, pp. 1636-1642, 2001.

[22] D. M. McDonald, G. Coleman, A. Bhatwadekar, T. A. Gardiner, and A. W. Stitt, "Advanced glycation of the Arg-Gly-Asp (RGD) tripeptide motif modulates retinal microvascular endothelial cell dysfunction," Molecular Vision, vol. 15, pp. 1509-1520, 2009.

[23] T. A. Gardiner, H. R. Anderson, and A. W. Stitt, "Inhibition of advanced glycation end-products protects against retinal capillary basement membrane expansion during long-term diabetes," Journal of Pathology, vol. 201, no. 2, pp. 328-333, 2003.

[24] U. Andersson and K. J. Tracey, "HMGB1 is a therapeutic target for sterile inflammation and infection," Annual Review of Immunology, vol. 29, pp. 139-162, 2011.

[25] R. Zhou, A. Tardivel, B. Thorens, I. Choi, and J. Tschopp, "Thioredoxin-interacting protein links oxidative stress to inflammasome activation," Nature Immunology, vol. 11, no. 2, pp. 136-140, 2010 . 


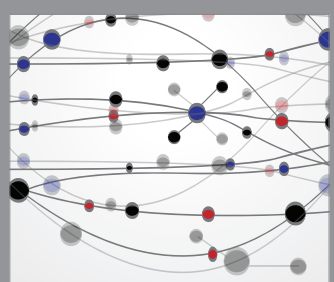

The Scientific World Journal
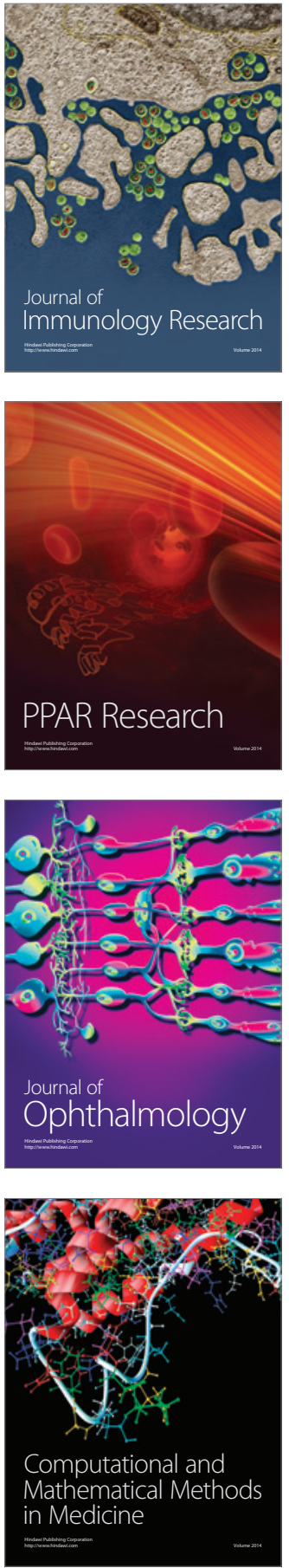

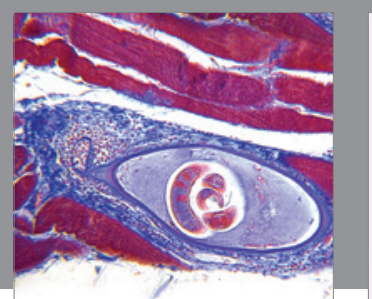

Gastroenterology Research and Practice
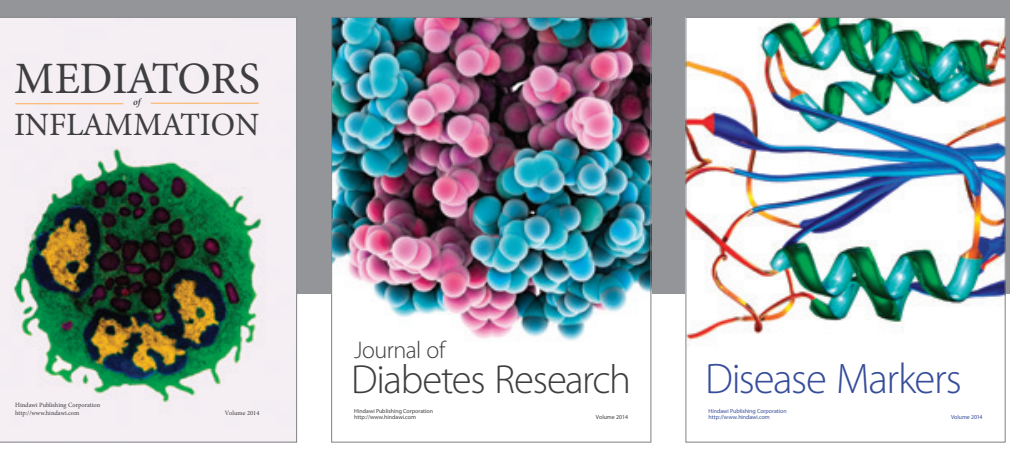

Disease Markers

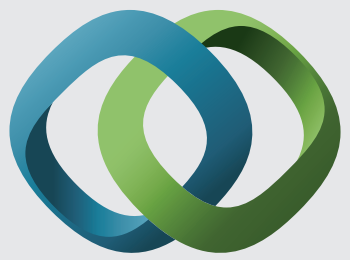

\section{Hindawi}

Submit your manuscripts at

https://www.hindawi.com
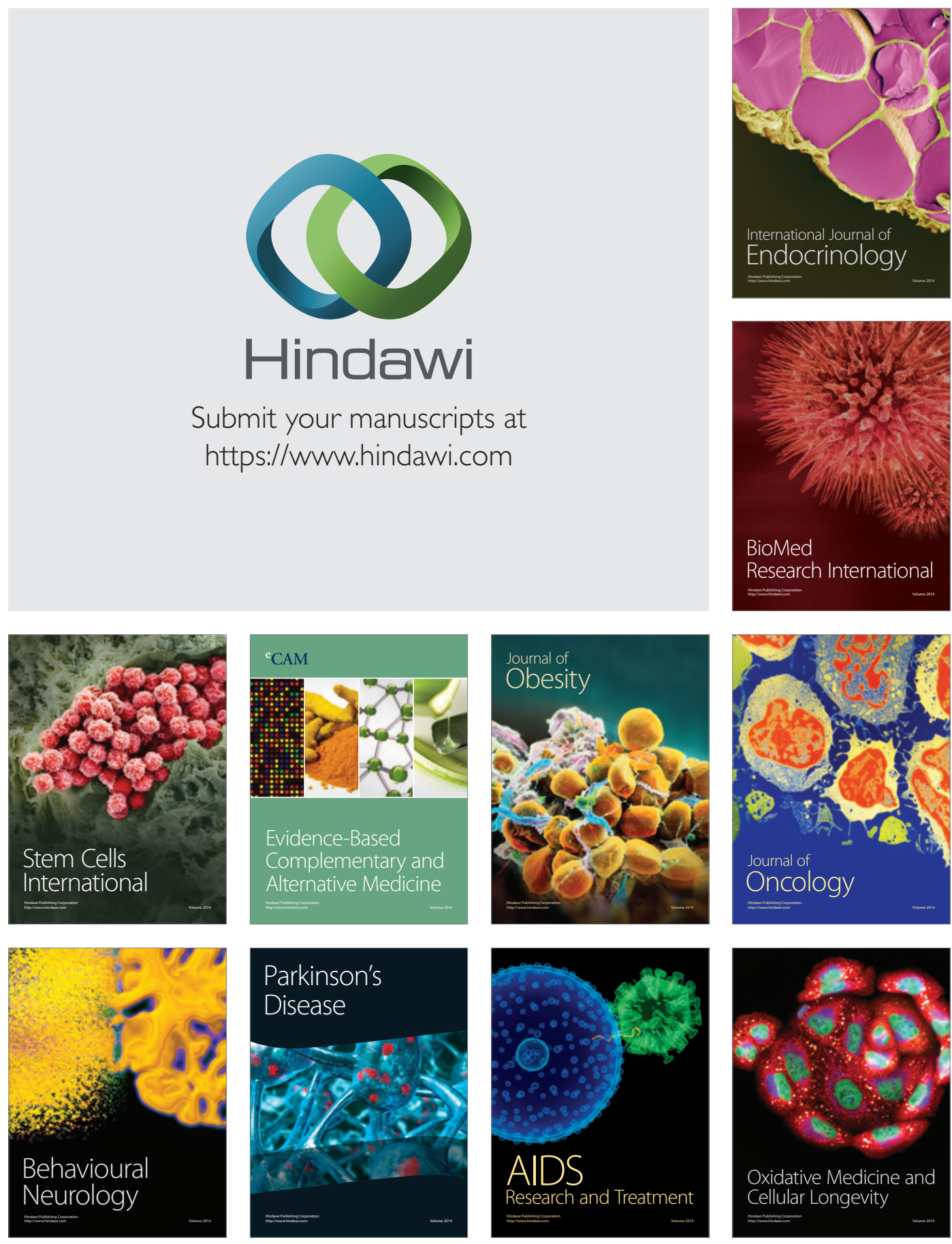